\title{
Iconographic representation of a dioptra in a flemish tapestry of the XV century
}

\section{Representación iconográfica de una dioptra en un tapiz flamenco del siglo XV}

\author{
Sergio Barbero $\left.{ }^{*}, \mathrm{~S}\right)$ \\ Instituto de Óptica (CSIC), Serrano 121, 28006, Madrid, Spain \\ ${ }^{*}$ Email: sergio.barbero@csic.es \\ S: miembro de SEDOPTICA / SEDOPTICA member \\ Received / Recibido: 18/11/2013. Revised / Revisado: 28/01/2014. Accepted / Aceptado: 30/01/2014. \\ DOI: http://dx.doi.org/10.7149/OPA.47.1.51
}

\begin{abstract}
:
Dioptra was an optical instrument widely used in Antiquity and later in the Middle Ages. In spite of the existence of different types of dioptra, all of them have the property of helping to fix the line of sight. The so-called sighting-tube, basically an empty tube to look through, was one of the simplest types of dioptra. In the history of art there are few reported iconographic representations of this type of instrument. However, such representations are of great historical value because they help to shed light on the possible uses and types of dioptra used during these times. The hypothesis established in this article is that we have discovered such a one representation, non-previously described, at a XV century Flemish tapestry. This tapestry, called "Holly Cross Captivity", is located in La Seo Chapterhouse Tapestry Museum in Zaragoza (Spain).
\end{abstract}

Key words: Dioptra, Sighting Tube, History of Optics, History of the Telescope, Flemish Art.

\section{RESUMEN:}

La dioptra era un instrumento óptico ampliamente utilizado en la Antigüedad y posteriormente en la Edad Media. A pesar de existir diversas variantes de dioptras, el denominador común de todas ellas era la propiedad de facilitar la fijación de la dirección de mirada. El tubo de mirada, en esencia un tubo hueco a través del cual se miraba, era uno de los tipos de dioptra más sencillos. En la historia del arte existen pocas representaciones iconográficas documentadas de este tipo de instrumentos. Tales representaciones son de gran interés histórico ya que permiten profundizar en el conocimiento de los posibles usos y tipologías de las dioptra utilizadas en estas épocas. La hipótesis de este artículo es que hemos descubierto una de estas representaciones, no descrita con anterioridad, en un tapiz flamenco del siglo XV. El tapiz en cuestión, denominado "Cautividad de la Santa Cruz", se encuentra alojado en el Museo de Tapices de la Seo de Zaragoza.

Palabras clave: Dioptra, Tubo de Mirada, Historia de la Óptica, Historia del Telescopio, Arte Flamenco.

\section{REFERENCES AND LINKS / REFERENCIAS Y ENLACES}

[1]. L. Spillmann, J. Hardy, P. Delahunt, B. Pinna, J. S. Werner, "Brightness enhancement seen through a tube", Perception 39, 1504-1513 (2010). DOI

[2]. R. K. G. Temple, El Sol de Cristal: Tecnologías Perdidas de la Antigüedad, Oberon, Madrid (2000).

[3]. M. J. T. Lewis, Surveying Instruments of Greece and Rome, Cambridge University Press, New York (2001).

[4]. A. J. Cappeletti, La Teoría Aristotélica de la Vision, Sociedad Venezolana de Ciencias Humanas, Caracas, Venezuela (1977).

[5]. S. Barbero, "Los defectos ópticos de la visión explicados por Aristóteles", Asclepio 65, 1-10 (2013). 
[6]. A. Jones, "Peripatetic and Euclidean theories of the visual ray", Physis Riv. Int. Stor. Sci. 31, 47-76 (1994).

[7]. C. Plug, H. E. Ross, "Historical review of the moon illusion", in The Moon Illusion, M. E. Hershenson, Lawrence Erlbaum Associates, Hillsdale (1989).

[8]. Strabo, Geografía, Libros III-IV. Editorial Gredos, Madrid (1992).

[9]. A. C. Bowen, R. B. Todd, Cleomedes' Lectures on Astronomy: A Translation of The Heavens, University of California Press, Berkeley (2004).

[10]. Galeno, Sobre la Utilidad de las Partes del Cuerpo Humano en Diecisiete Libros, Ediciones Clásicas, Madrid (2009).

[11]. D. W. Hughes, "On seeing stars (especially up chimneys)", Q. J. Roy. Astronom. Soc. 24, 246-257 (1983).

[12]. A. G. Smith, "Daylight visibility of stars from a long shaft", J. Opt. Soc. Am. 45, 482-483 (1955). DOI

[13]. G. Galilei, Diálogo sobre los Dos Máximos Sistemas del Mundo Ptolemaico y Copernicano, Alianza Editorial, Madrid (2011).

[14].E. Reeves, "Complete inventions: The mirror and the telescope", in The Origins of the Telescope, KNAW Press, Amsterdam (2010).

[15]. D. L. Couprie, Heaven and Earth in Ancient Greek Cosmology: From Thales to Heraclides Ponticus. Springer, New York (2011). DOI

[16]. J. Evans, The History and Practice of Ancient Astronomy Oxford University Press, New York (1998).

[17]. H. C. King, The History of the Telescope, Sky Pub. Corp, Cambridge, Mass (1955).

[18]. E. T. P. Ortiz, Sobre las Líneas Indivisibles; Mecánica, Gredos, Madrid (2000).

[19]. Vitruvio, Los Diez Libros de Arquitectura. Introducción de Delfín Rodríguez Ruiz. Traducción de José Luis Oliver Domingo. Alianza Editorial, Madrid (1995).

[20]. A. M. Smith, Ptolemy's Theory of Visual Perception: An English Translation of the Optics, American Philosophical Society, Philadelphia (1996).

[21]. D. J. Price, Precision Instruments: To 1500 in A History of Technology. Vol. 3, From the Renaissance to the Industrial Revolution, c.1500-c.1750, Clarendon Press, Oxford (1957).

[22]. J. J. Coulton, "The dioptra of Hero of Alexandria", in Science and Mathematics in Ancient Greek Culture, T. E. Tuplin, Edt., Oxford University Press, Oxford (2002). DOI

[23]. D. Sperber, Nautica Talmudica, Bar-Ilan University Press, Leiden (1986).

[24]. J. Needham, L. Wang, Science and Civilisation in China. Volume 3, Mathematics and the Sciences of the Heavens and the Earth Cambridge University press, Cambridge (1959).

[25]. R., Eisler, "The polar sighting tube", Archives Internationales d'Historie des Sciences 2, 312-332 (1949).

[26]. H., Michel, "Les tubes optiques avant le télescope", Ciel et Terre 70, 175-184 (1954).

[27]. Iamblichus, J. M. Dillon, J. P. Hershbell, On the Pythagorean Way of Life. Scholars Press, Atlanta, Ga (1991).

[28]. E. A. Torra, Hombría, T. Domingo, Los Tapices de La Seo de Zaragoza, Editorial C.A.I., Zaragoza (1985.)

[29].É. Bertaux, Exposición Retrospectiva de Arte -1908, Tip, La Editorial, Zaragoza (1910).

[30]. M. A. Zalama, J. Molina, "Tapices de Juan II de Aragón y Fernando el Católico en La Seo de Zaragoza". Boletín del Museo e Instituto Camón Aznar 109, 285-320 (2012).

[31].S. Vorágine, La Leyenda Dorada II. Traducción de José Manuel Macías. Alianza Editorial, Madrid (2005).

\section{Introducción}

El tubo de mirada (sighting-tube en la bibliografía anglosajona) era un instrumento óptico que se utilizaba en la Antigüedad y en la Edad Media, sobre el cual siguen existiendo numerosos interrogantes, siendo el principal saber si eran tubos huecos, o si en cambio en su interior disponían de algún tipo de lente óptica. En este último caso el tubo de mirada sería un claro precursor del telescopio, sino un telescopio en sí. Sin embargo lo más aceptado es que eran realmente tubos huecos. Lo que es seguro es que se trataba de un instrumento utilizado para 
mirar a través de él, y cuya función era, de alguna manera, mejorar la visión de determinados objetos, principalmente celestes.

El fundamento físico del tubo hueco, cómo ayuda visual, radica en el hecho de que objetos poco brillantes pueden observarse mejor cuando luces espurias provenientes de otros lugares del campo visual son bloqueadas por la apertura del tubo. Recientes estudios psicofísicos [1] han demostrado que cuando se mira con un ojo a través de un tubo, a la vez que el otro ojo se mantiene abierto, se produce una sensación de incremento de percepción del brillo en el ojo que mira a través del tubo.

El tubo de mirada pertenece a una familia de instrumentos a la que se denominaba dioptra. Esta palabra griega significa literalmente: algo a través de lo cual mirar. El origen etimológico de la palabra dioptra ha sido analizado en [2-3]. Las diferencias entre las distintas dioptra quedan de manifiesto por la variedad de representaciones iconográficas, fundamentalmente de la Edad Media, aunque también se ha documentado una representación procedente de la antigüedad clásica (Fig. 1). Diversas referencias clásicas hacen mención más o menos indirecta a estas dioptra. En este artículo, si bien no aportamos ninguna tesis novedosa sobre este tipo de instrumentos, sí presentamos una revisión crítica sobre lo que se sabe sobre ellos.

Por lo dicho, la prueba más fehaciente de la existencia de los tubos de mirada es su identificación en representaciones iconográficas en la historia del arte. Ya que no son muchas las representaciones encontradas - de las cuales haremos un repaso - éstas son de especial relevancia para entender mejor con qué fines se utilizaban estos instrumentos.

El objetivo de este artículo es tratar de demostrar que hemos encontrado una representación - quizás la primera que se documenta en España - de una dioptra en el arte medieval. En concreto la susodicha representación aparece en un tapiz flamenco medieval que actualmente se expone en el Museo de Tapices de la Seo de Zaragoza.

El artículo está estructurado en dos secciones. En primer lugar se hará una

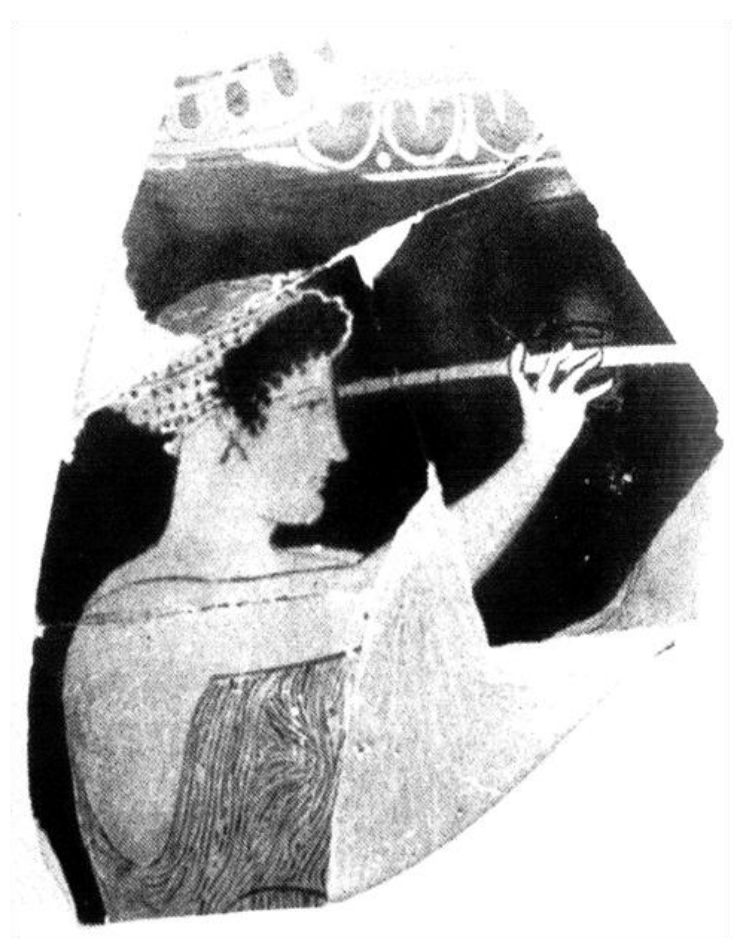

Fig. 1. Tubo de mirada de tipo cónico. Fotografía de fragmento de vasija griega del siglo V-IV a.C. Reproducido por Temple [2].

exposición crítica del estado del arte del conocimiento historiográfico sobre las dioptra y, en particular, sobre los tubos de mirada. En segundo lugar se describirá el tapiz encontrado, y se justificará nuestra hipótesis interpretativa.

\section{La dioptra en la antigüedad}

Dentro del esquema de la teoría de la visión aristotélica [4], la visión se produce por una actualización (transformación en acto) de la potencia del medio presente entre el objeto visible y los ojos. Este medio, potencialmente diáfano, se transforma en transparente gracias a la acción de la luz. Cuando el medio es en acto transparente, la visión deviene posible. Esta transformación se produce de manera instantánea, siendo más intensa cuanto más concentrado es el medio. De esta manera, si se redujese el espacio entre el objeto visible y los ojos, la agudeza visual mejoraría. Por ejemplo, los ojos más hundidos dispondrían de mayor agudeza. Por lo tanto, la agudeza visual está correlacionada con la morfología del ojo [5]. De aquí que Aristóteles llegue a una conclusión 
especialmente relevante para nuestro estudio de las dioptra:

En efecto, la misma persona que haciéndose sombra con la mano sobre los ojos o mirando a través de un

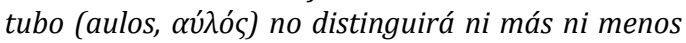
las diferencias de los colores, sin embargo, verá más lejos. Algunos, al menos, ven a veces las estrellas desde zanjas y pozos. (Reproducción de los animales $V, 780 b 15-25)$

De hecho, se verían especialmente bien las cosas distantes, si hubiera una especie de tubo (aulos) continuo desde el ojo hasta el objeto contemplado, pues no se perdería el movimiento procedente de los objetos visibles; pero si no es así, cuanto más lejos se extienda el tubo, con más precisión se verán necesariamente los objetos alejados. (Reproducción de los animales $V, 781$ a 7-10).

La teoría de la visión aristotélica aceptaba el concepto de cono visual; esto es, el objeto sensible y el ojo forman un cono, de manera que sólo lo localizado dentro de este cono visual es visto. De aquí se deduce que el tubo simplemente serviría para reducir el ángulo del cono visual [6], y por tanto concentrar la fuerza visual en una región concreta. De hecho veremos como la representación de tubos de mirada de forma cónica podría tener relación con este concepto geométrico del cono visual, el cual no era patrimonio exclusivo de Aristóteles, sino que era ampliamente utilizado por otras corrientes de pensamiento, en especial la tradición de óptica matemática griega.

Conviene señalar que la palabra griega aulos tenía otra acepción. Denotaba un típico instrumento musical de viento que era una especie de oboe doble, formado por dos tubos cónicos con una seria de agujeros. La Fig. 2, muestra una escultura de un sátiro tocando un oboe. Si esto indica que el instrumento musical aulos fue también utilizado, siquiera ocasionalmente, como instrumento de mirada sólo puede ser una especulación, aunque una plausible.

También a Aristóteles le debemos la primera explicación de la llamada "ilusión lunar" fenómeno por el cual la Luna (o el Sol) parece tener un tamaño mayor cuando se ve en el horizonte respecto a cuando se ve en el cenit [7]. La explicación aparece recogida en su tratado Meteorológicos, donde se describe el fenómeno como consecuencia de una desviación de «los rayos visuales» al atravesar medios con humedad (lo que hoy en día se denomina refracción atmosférica). En estos casos la visión del astro aparece magnificada.

El efecto magnificador de los medios húmedos y la visión a través de los aulos aparecen relacionados en un texto de Estrabón (al citar una observación de Posidonio sobre el crespúsculo): «en los mares aumenta la apariencia de su tamaño [el sol], lo mismo en las puestas que en las salidas, porque los vapores se elevan de las aguas en gran cantidad: la vista, quebrándose a través de ellos como a través de aulos recibe las imágenes ampliadas»[8]. Lo interesante de este fragmento radica en que se sugiere un efecto magnificador de los aulos, lo cual ha servido de argumento a los historiadores que preconizan estos aulos, como auténticos telescopios.

Posidonio de Apamea (c. 135 a. C.-51 a. C.) pertenecía a la escuela de los estoicos. Para los estoicos la visión se producía por la acción de una sustancia llamada pneuma, de naturaleza mixta ígnea y aérea, que permitía la aprehensión del objeto sensible mediante un cambio en la tensión del medio envolvente. Los estoicos también aceptaban la noción de cono visual. El medio circundante afectado por el pneuma formaba un cono con vértice situado en el órgano sensorial y base en el objeto sensible. Ahora bien el movimiento del pneuma, y por tanto la percepción visual, puede verse afectada por la densidad del aire circundante.

El estoico Cleomedes, en un interesante tratado sobre astronomía (Caelestia) escrito en alguna fecha entre el año 50 a. C. y 250 d. C., argumenta que el sol se ve más grande desde el fondo de un pozo porque el pneuma visual se ve afectado al atravesar una zona con mayor humedad o densidad [9]. Relacionado con esto, se encuentra la extendida creencia de la Antigüedad, mantenida a lo largo de la Edad Media, de que las estrellas pueden ser observadas durante el día si nos encontramos dentro de un pozo. Galeno, por ejemplo, lo afirma en su Sobre la utilidad de las partes del cuerpo humano: «Pero también desde los pozos profundos se ven las estrellas, sobre todo cuando el sol no está en su mediodía» [10]. Sin embargo se sabe que esto no es posible [11,12]. 


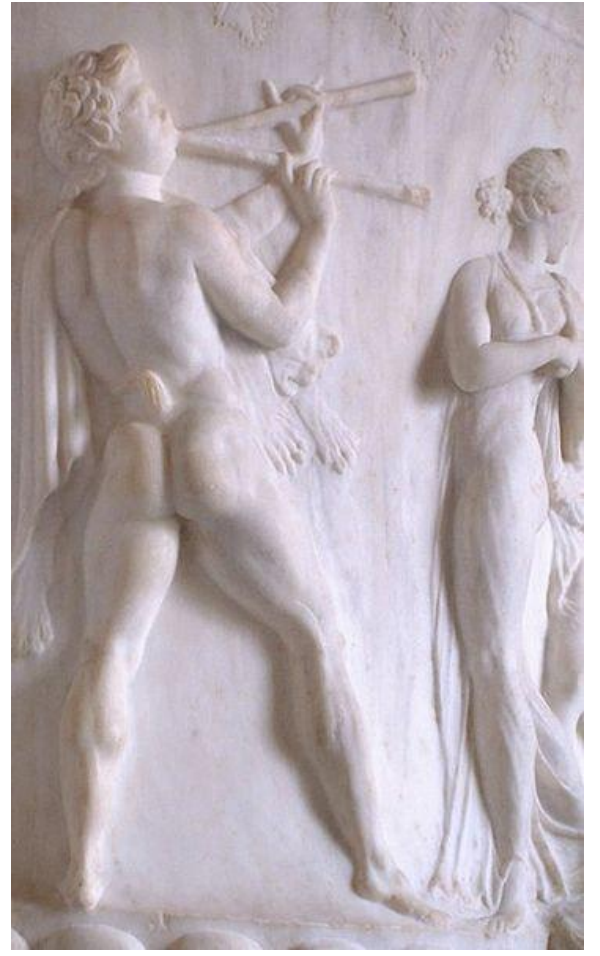

Fig. 2. Instrumento musical denominado aulos. Escultura griega, representado a un sátiro, localizada en el Museo del Louvre. Imagen extraída de commons.wikimedia.org.

De hecho, Galileo Galilei en su Diálogo sobre los dos principales sistemas del mundo (1632) $[13,14]$, en boca de Salviati, llegó a afirmar que la idea original inherente al descubrimiento del telescopio radica precisamente en esta supuesta observación:

Todavía viven y gozan de buena salud algunos gentilhombres que estuvieron presentes cuando un doctor, profesor de una universidad famosa, al oír describir el telescopio que él todavía no había visto, dijo que la invención estaba tomada de Aristóteles. $Y$ habiéndose hecho traer un texto, encontró cierto lugar donde se explica a qué se debe el que desde el fondo de un pozo muy hondo, de día se puedan ver las estrellas del cielo. Y dijo a los que le rodeaban: "He aquí que el pozo denota el tubo. He aquí los vapores groseros, de los que se ha sacado la invención de los cristales. $Y$ en definitiva he aquí, reforzada, la vista al pasar los rayos por el diáfano más denso y oscuro».

\section{2.a. Los dioptra como instrumento de medida}

Las primeras observaciones astronómicas se realizaban simplemente con los ojos y sin ningún tipo de instrumento de ayuda visual. Más tarde, se empezó utilizar el propio cuerpo para realizar medidas tales como distancias angulares. Los primeros instrumentos artificiales, creados por el ser humano, pudieron ser tablas de medidas conocidas cuyo fin era comparar distancias angulares [15] o el gnomon (un objeto vertical, como un palo, en el cual se observa la sombra proyectada) [16].

Otro de los mecanismos de observación más antiguos era el denominado merkhet, utilizado por los egipcios. El sistema consistía en marcar una línea imaginaria mediante dos objetos a través de los cuales la mirada debía estar dirigida. Estos dos objetos o bien podían ser dos plomadas, o una plomada y un visor (por ejemplo una hoja de palma partida) $[3,17]$ (ver las Figs. 1 y 2 reproducidas en [17]). Así, para fijar la dirección norte-sur, la línea de mirada podría dirigirse a la estrella polar.

La primera referencia escrita $[3,16]$ sobre una dioptra, como instrumento de medida, aparece en un tratado de astronomía (Fenómenos), cuya autoría se adscribe a Euclides. En la primera proposición del tratado se enseña que la tierra se sitúa en el centro de la esfera celeste (el cosmos). Para su justificación se describe cómo la constelación de cáncer, la de capricornio y la tierra se encuentran en línea recta [18]. Esto se puede observar con ayuda de una dioptra. Sin embargo, de esta descripción no se puede deducir como era exactamente la dioptra de Euclides. Podría ser un sistema parecido el merkhet egipcio, un mero soporte con dos orificios para delimitar una línea recta con la mirada, o un tubo de mirada.

Lamentablemente, a pesar de que el uso de dioptras en la antigüedad está muy documentado, no se conserva ningún resto arqueológico de ninguna dioptra, aunque en una reciente revisión sobre los orígenes del telescopio Eileen [14] recoge la cita de un jesuita (Liberto Froidmont) quien en un tratado de 1627 (Meteorologica) describía el hallazgo en un castillo de Hainault de: «un tubo dióptrico (dioptricus tubus), muy oxidado y antiguo». Conviene advertir, sin embargo, que por dioptra se denotaban una gran diversidad de instrumentos, desde el más sencillo compuesto de una barra con dos orificios, un tubo vacío, a un instrumento de la complejidad del "dioptra compuesto" descrito por Geminus (véase [16]). En cualquier caso todos ellos comparten el 
denominador común de ser un sistema para fijar una línea de mirada.

El historiador griego Polibio (siglo II a. C.) describe en sus historias un sistema de telegrafía óptica con fines militares. En un extremo se situaba una persona que realizaba señales codificadas con la ayuda de dos antorchas; en el otro extremo del telégrafo óptico, el receptor observa las señales con una dioptra, compuesta de dos tubos, cada uno en línea con cada una de las antorchas.

Marco Vitrubio (siglo I a. C.) describió la dioptra como un instrumento para medir desniveles del terreno, aunque advierte que dicha técnica tenía errores y recomienda el uso de otros sistemas de medida más fiables: «El nivel se fija con la ayuda de la dioptra, con niveles de agua, o bien con un corobate. El mejor método es usar un corobate, pues la dioptra y los niveles de agua fallan en ocasiones» [19]. Olimpiodoro el Joven (fl. siglo VI a. C.) proporciona una explicación del funcionamiento de las dioptra en concordancia con las nociones Aristotélicas y estoicas de la concentración visual (recogido en [3]): «Las dioptras fueron inventados por los mecánicos para que la vista fuese dirigida a través de un pasaje estrecho y por tanto no se dispersase». En este pasaje, dioptra, parece denotar un tubo de mirada.

También existen referencias al uso científico de dioptras. Ptolomeo se valió de un dispositivo - que probablemente consistía de un orificio de mirada montado sobre un soporte rotatorio [20] - para medir el ángulo de reflexión de la luz en un espejo. Según escribe Ptolomeo en su Almagesto Hiparco inventó un instrumento llamado dioptra, que utilizó para medir el diámetro aparente del sol y de la luna. Posteriormente Pappus de Alejandria [21] describió esta dioptra como un sistema compuesto por una barra con una ranura, un prisma y unas placas con orificios. Una figura reconstruida del tal instrumento aparece en [21]. Un claro precursor de este tipo de dioptra, a pesar de ser un sistema más simple que el descrito por Ptolomeo, es el utilizado por Arquímedes de Siracusa en su obra El contador de arena para medir el tamaño del sol. Sosigenes (ca. 165), filósofo peripatético, usó aparentemente un instrumento similar al tratar de medir el tamaño aparente de la luna. También Gemino describe en un libro de introducción a la astronomía (Introducción a los fenómenos) el uso de una dioptra.

Debido a diversos factores, ya en tiempos de Ptolomeo la dioptra, como instrumento de medida de la posición de las estrellas, empezó a ser sustituido por la esfera armilar [16]. En cambio, en topografía terrestre, seguiría evolucionando llegando a su clímax con la dioptra aparentemente diseñada por Herón de Alejandría. Afortunadamente nos ha llegado una descripción detallada, aunque incompleta por faltar ocho páginas del tratado, de este sistema en su tratado sobre topografía terrestre denominado precisamente Dioptra (c. 62 d.C.) [3]. El instrumento descrito por Herón es en esencia un teodolito [22], es decir un sistema mecánico-óptico para medir las coordenadas esféricas de una dirección de mirada.

Aparte de las referencias a la civilización griega la dioptra, en su variante de tubo de mirada, también fue usada por otras civilizaciones antiguas. En algunos documentos talmúdicos se ha documentado el uso de un tubo denominado sefoforet que era utilizado para mirar a través de él (mezupit) [3]. El rabino Gamaliel II (80 d.C) utilizaba este tubo de mirada para saber cuando un barco se encontraba situado a determinada distancia (información necesaria para ciertas prescripciones del Sabbath). Es interesante resaltar que, de manera paralela a lo que ocurre con el término aulos, la palabra hebrea mezupit, era también usada para denotar un instrumento musical de viento en forma de trompeta [23].

Los tubos de mirada también aparecen ampliamente documentados en la civilización china [24]; tanto en astronomía - por ejemplo en un tratado sobre matemática se menciona que tal dispositivo se intentó usar para medir el diámetro del sol [24] - como en topografía (Fig. 3).

\section{Iconografía de los tubos de mirada}

Robert Eisler [2] fue el primero en analizar la aparición de este tipo de instrumentos en la iconografía. Poco tiempo más adelante Henri 


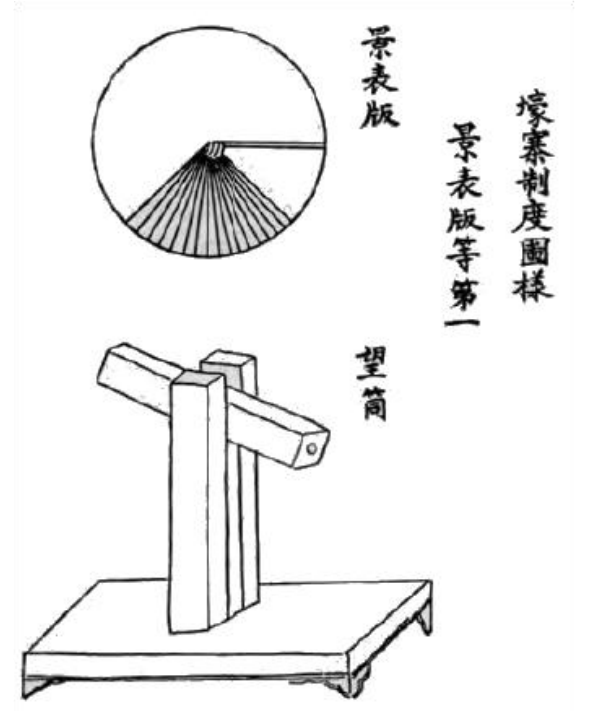

Fig. 3. Tubo de mirada y cuadrante utilizado por un arquitecto chino recogido en el tratado sobre arquitectura Ying Tsao Fa Shih (1103). Reproducido por [24].

Michel [26] clasificó estas representaciones en dos tipos diferentes. Los que, siguiendo a Eisler, denominó «tubos polares», utilizados como instrumento de medición astronómica, y los que en cambio no pueden tener tal función. Por otro lado Derek J. Price [21] clasificó los tubos en dos grupos. En el primero el tubo de mirada se encuentra integrado en un sistema mecánico, el cual permite fijar con estabilidad la dirección de mirada. Este tipo de montaje tendría claramente una finalidad astronómica. El segundo grupo, tubos de forma cónica (o tipo trompeta), serian tubos directamente sujetados con las manos delante de los ojos. Price sugirió que este segundo tipo de tubo, en forma de embudo, vendría influido por las nociones sobre la visión mantenidas por Aristóteles.

\section{3.a. El tubo polar}

La más conocida ilustración - primero documentada por Eisler [25] -, sobre este tipo de instrumento, aparece en una miniatura de un manuscrito de los siglos X-XI proveniente de la biblioteca de la Abadía benedictina de San Galo (Suiza) acompañando un texto de Gerberto de Aurillac (quien fuera papa Silvestre II). La Fig. 4 ilustra a un astrónomo mirando a través de un largo tubo. Éste se sujeta sobre una columna de estilo corintio, de manera que el ángulo de mirada se mantiene fijo.

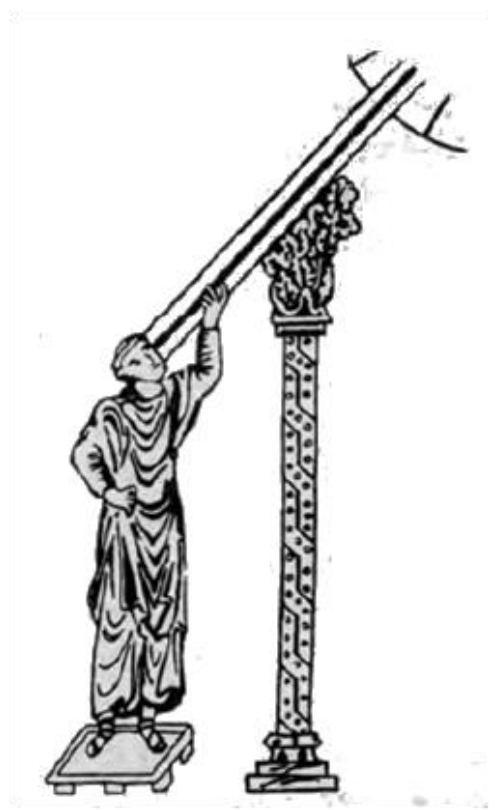

Fig. 4. Tubo de mirada para observar la estrella polar. Recogido en una miniatura de un manuscrito medieval del siglo X. Reproducido por $[25,26]$.

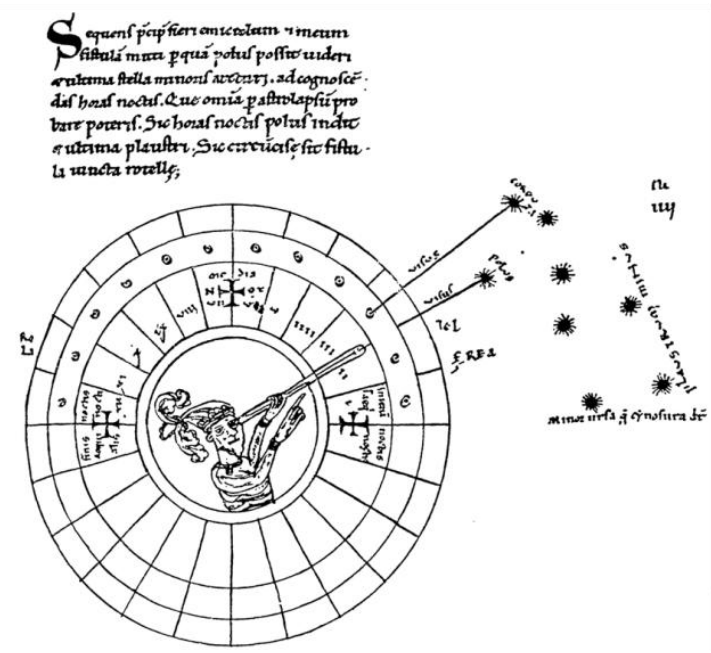

Fig. 5. Tubo de mirada para observar la estrella polar. Recogido en un manuscrito medieval del siglo XII. Reproducido por [26].

Gerberto describe de manera precisa el funcionamiento del tubo de mirada para identificar si una estrella es la estrella polar o no. En primer lugar se fija la posición del tubo de manera que se vea la estrella a analizar en un momento dado. Si esta estrella permanece visible en la línea de mirada definida por el tubo y a lo largo de la noche, entonces se puede afirmar que la estrella es la estrella polar [26]. 
Otra ilustración (Fig. 5), que se puede caracterizar cómo tubo polar, fue descubierta por Michel [26] en un manuscrito del siglo XII en la Biblioteca de Chartres. De nuevo un astrónomo mira a través de un tubo a la estrella polar. El semicírculo graduado sirve para fijar la hora de la noche a la que se realiza la observación.

\section{3.b. Los tubos cónicos}

Robert Temple [25] ha descubierto una representación de especial relevancia, por ser la única de la antigüedad griega documentada hasta la actualidad, pintada en un fragmento de vasija rota expuesto en el museo de la Acrópolis de Atenas y que puede datarse del siglo V-IV a.C. (Fig. 1). La vasija muestra a una persona que mira a través de un tubo de forma cónica.

Siguiendo un orden cronológico, la siguiente figura (Fig. 6), muestra a un astrónomo observando una estrella a través de un tubo cónico. El fin debería justificar el clasificar este tubo de mirada dentro de los tubos polares. Sin embargo, el hecho de que el astrónomo sujeta el tubo con su mano, lo desacredita, según Michel y Temple. Desde nuestro punto de vista, lo determinante es el hecho que el tubo es de forma

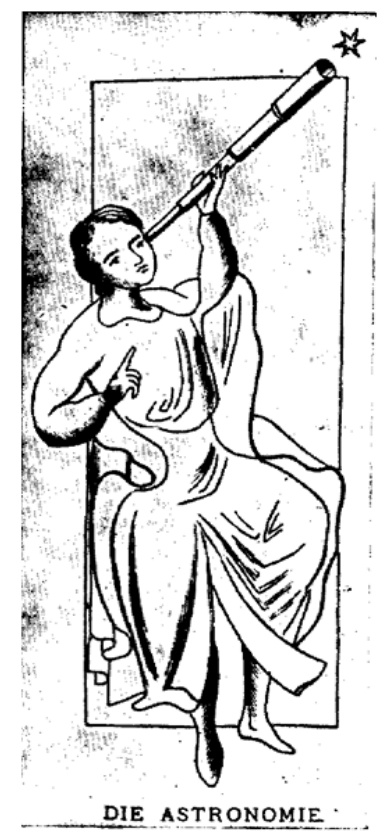

Fig. 6. Tubo de mirada de forma cónica. Representación de un astrónomo recogida en un manuscrito medieval del siglo XIII. Reproducido por $[25,26]$. cónica. Otra peculiaridad de esta ilustración es que se ve que el tubo es extensible. La ilustración, que aparece en un manuscrito medieval del siglo XIII (Fig. 7 [25]), es parte de un motivo iconográfico muy habitual de la Edad Media: la personificación de los artes medievales (Trivium y Quadrivium). En este caso la figura personifica a la astronomía (parte del Quadrivium).

El último ejemplo documentado de tubo cónico (Fig. 7) aparece en un manuscrito del siglo XV (reproducido cómo Fig. 6 en [25], véase también [26]). La figura representa a Pitágoras y a Sócrates. De manera alegórica se contrapone la astronomía - representada por Pitágoras observando el cielo - y la geometría - con un Sócrates realizando medidas con ayuda de un compás y una escuadra. Jámblico relata cómo Pitágoras hacía uso de la dioptra para ayudar a la vista [27]: «En cierta ocasión, sumergido en profundo pensamiento, cavilaba sobre cómo podría inventar algún instrumento de ayuda al oído, estable y preciso, del mismo modo que la vista se sirve por medio de un compás, una regla, o de hecho un dioptra...». Esta representación es especialmente relevante, porque en el tapiz de la Cautividad de la Santa Cruz objeto de nuestro estudio, junto a la figura que porta la dioptra (Fig. 10) también hay otra figura que tiene entre manos una escuadra (Fig. 9).

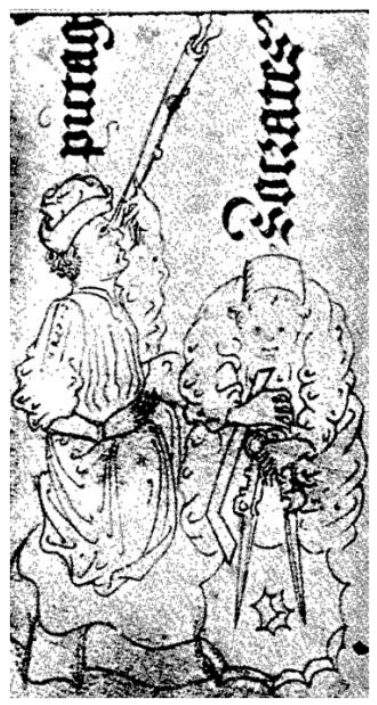

Fig. 7. Pitágoras mirando a través de un tubo de mirada. Recogido en un manuscrito medieval del siglo XV. Reproducido por [25]. 


\section{El tapiz de la Cautividad de la Santa Cruz}

Tras la contextualización de las representaciones iconográficas documentas, trataremos de argumentar la existencia de una representación de una dioptra en un tapiz flamenco del siglo XV.

El tapiz en cuestión, denominado "Cautividad de la Santa Cruz", se encuentra alojado en el Museo de Tapices de la Seo de Zaragoza ${ }^{1}$. Este tapiz destaca por sus grandes dimensiones $\left(406 \times 1008 \mathrm{~cm}^{2}\right)$. En conjunción con el denominado "Exaltación de la Santa Cruz" (el cual en la actualidad no se expone) forman una serie común "Exaltación de la Santa Cruz" que representan la Historia del rey persa Cosroes Cosroes II reinó entre 590 y 628 d.C. Fotografías a color y de alta resolución de ambos tapices se pueden encontrar en [28].

Son realmente dos de los tapices más valiosos de toda la colección del Cabildo zaragozano, siendo en palabras de Emile Bertaux: «las únicas representaciones de la leyenda de la Cruz que conocemos en el arte flamenco» [29]. Su confeccionado, fechado en torno a la segunda mitad del siglo $\mathrm{XV}$, siguen el peculiar estilo de trenzado de hilos de Tournai (situado en la Bélgica actual) [28].

Tal como aparece documentado en el «Inventario de la Sacristía Mayor de La Seo», conservado en el Archivo Capitular de la Seo de Zaragoza, los tapices de la Exaltación de la Santa Cruz llegaron a la Seo procedentes del monasterio jerónimo de Santa Engracia de la misma ciudad [28]:

Ay seis paños que se han traido de Sta. Engracia, los dos muy grandes, con la historia del rey Cosroas de Persia, de personados vestidos a lo antiguo, y unos rótulos góticos en campo blanco... Ay cinco paños de diferentes estofas e historias, que llaman de las espadas: eran estos seis paños, y el uno que falta, se dió por los de Sta. Engracia, con quatro paños más llamados de Ortiz.

\footnotetext{
${ }^{1}$ La colección de tapices del Cabildo Metropolitano de Zaragoza, formada por más de sesenta tapices flamencos de los siglos XV, XVI y XVII, está hoy considerada una de las más importantes del mundo. Muchos de los paños fueron donados a la sede zaragozana en el siglo XVI por algunos arzobispos de la ciudad como Alonso de Aragón, (hijo del rey Fernando el Católico) o Andrés Santos.
}

De Santa Engracia los dos paños pasaron a la Seo de Zaragoza mediante un intercambio de tapices, aunque las motivaciones de la permuta son desconocidas ${ }^{2}$. Una reciente investigación ha documentado que estos dos tapices, y otros que el rey Católico entregó al cenobio jerónimo, habían pertenecido anteriormente al rey Juan II de Aragón, padre de Fernando el Católico[30].

\section{4.a. Análisis iconográfico}

Estos dos tapices siguen fielmente el texto que el dominico italiano Santiago de la Vorágine hace en su Leyenda Dorada, escrita en el siglo XIII, del episodio de la Exaltación de la Santa Cruz [31]. Ambas piezas narran el robo de la Santa Cruz en Jerusalén por el rey persa Cosroes y su posterior devolución a la ciudad santa por el emperador bizantino Heraclio.

El tapiz de la Cautividad representa cuatro escenas de la leyenda, con un orden de aparición de izquierda a derecha. Cada escena viene descrita por un texto en latín inscrito en la parte inferior del tapiz. Nos importa especialmente la tercera escena en la que Cosroes, sentado sobre su trono real y bajo la bóveda de una torre, recibe pleitesía y adoración. Una foto de esta escena se puede ver en la Fig. 8.

La inscripción en latín de esta escena reza lo siguiente $^{3}$ [28]:

Cosroes, rey de los persas, queriendo ser adorado como dios y que todos los judíos le diesen este título, colocó junto a sí la parte de la cruz que trajo de Jerusalén, en un trono artístico y precioso que había mandado construir.

Según la descripción de la Leyenda Dorada [31]:

Este monarca, en su afán de que sus súbditos le tuvieran por dios, hizo edificar una torre a base de oro, plata y piedras preciosas, colocó en el interior de la misma imágenes del sol, de la luna y de las estrellas, e instaló en las inmediaciones de la

\footnotetext{
${ }^{2}$ El monasterio de Santa Engracia fue fundado por el rey Fernando el Católico en 1493 en cumplimiento del testamento hecho por su padre Juan II según afirma el padre Sigüenza (1544-1606) en su Historia de la Orden de San Jerónimo.

${ }^{3}$ COSDROE, REX PERSARUM,VOLENS COLI UT DEUS ET SE DEUM AB OMNIBUS JUDEIS APPELLARI, PARTEM SANCTAE CRUCIS, QUAM DE JHERUSALEM ASPORTAVIT, IN THRONO QUEM ARTIFICIALITER ET PRECIOSISSIME EDIFICARE JUSSERAT JUXTA SE COLLOCAVIT.
} 


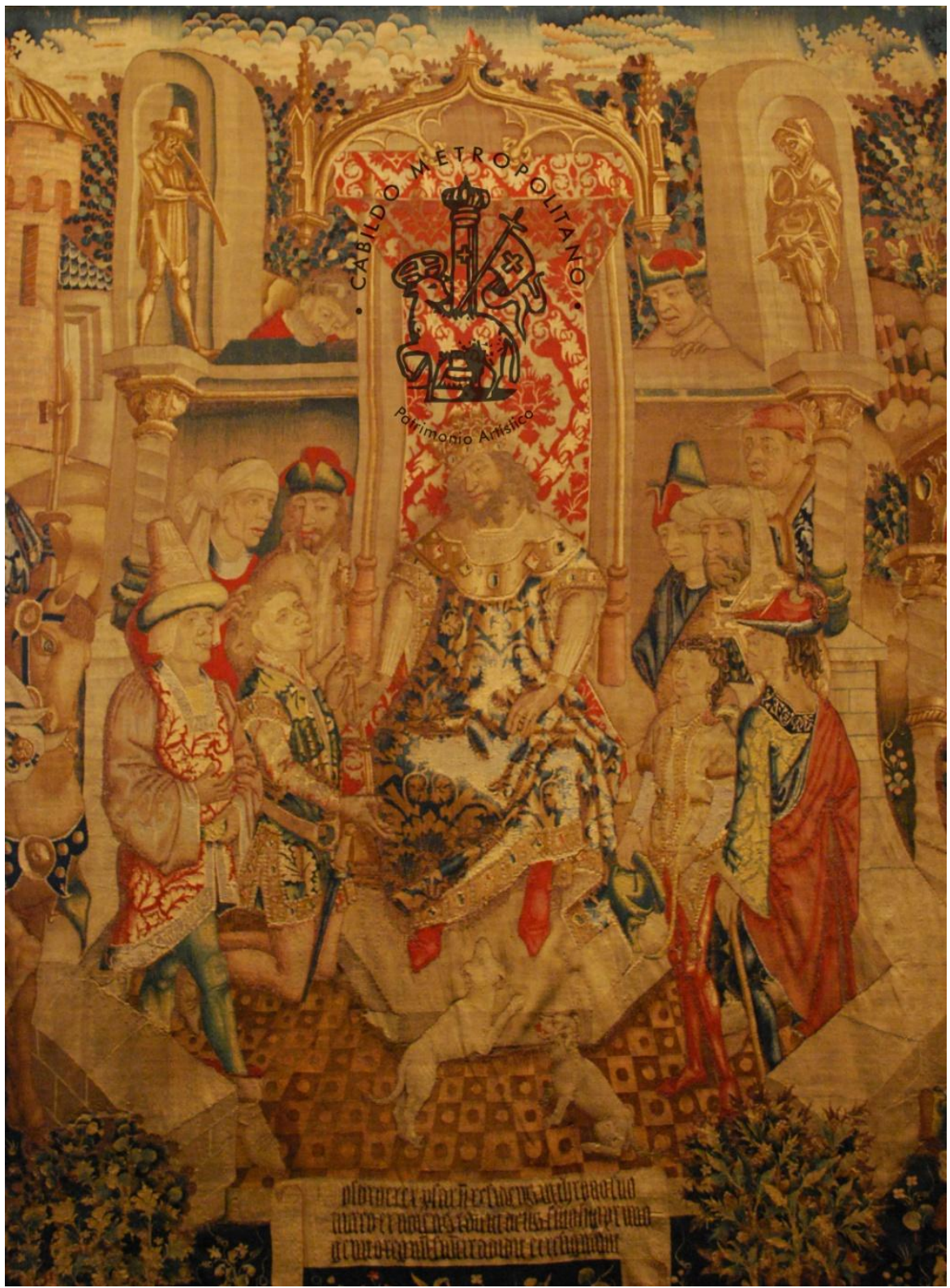

Fig. 8. Escena del Tapiz de "Cautividad de la Santa Cruz". Fotografía cedida por cortesía del Museo de Tapices del Cabildo Metropolitano de Zaragoza.

fortaleza ciertos dispositivos mecánicos ocultos, hábilmente construidos y de tal modo combinados, que mediante convenientes manipulaciones secretas dejaban caer desde lo alto agua en forma de lluvia sobre la torre. Con semejante procedimiento resultábale a Cosroas sumamente fácil convencer a la gente de que tenía poderes divinos [...]

Así pues, según la leyenda, Cosroes se ayudaba de ingenios y artificios técnicos para engañar a sus súbitos y hacerlos creer que tenía poderes divinos ${ }^{4}$. La técnica, y la ciencia, al

${ }^{4}$ Recuerda el mecanismo creado por Herón de Alejandría, descrito en su tratado Neumática, para abrir de manera servicio del sátrapa para embaucar al pueblo. En este marco conceptual se pueden interpretar las dos misteriosas figuras doradas que coronan la decoración escultórica del trono de Cosroes.

La figura de la derecha (Fig. 9) representa a un hombre que sujeta entre sus manos un aro y una escuadra (cf. Fig. 7). No podemos dejar de resaltar el parecido con la Fig. 7, donde también veíamos un hombre con una escuadra y, en su

automática las puertas de un templo, a la vez que sonaban varias trompetas. 


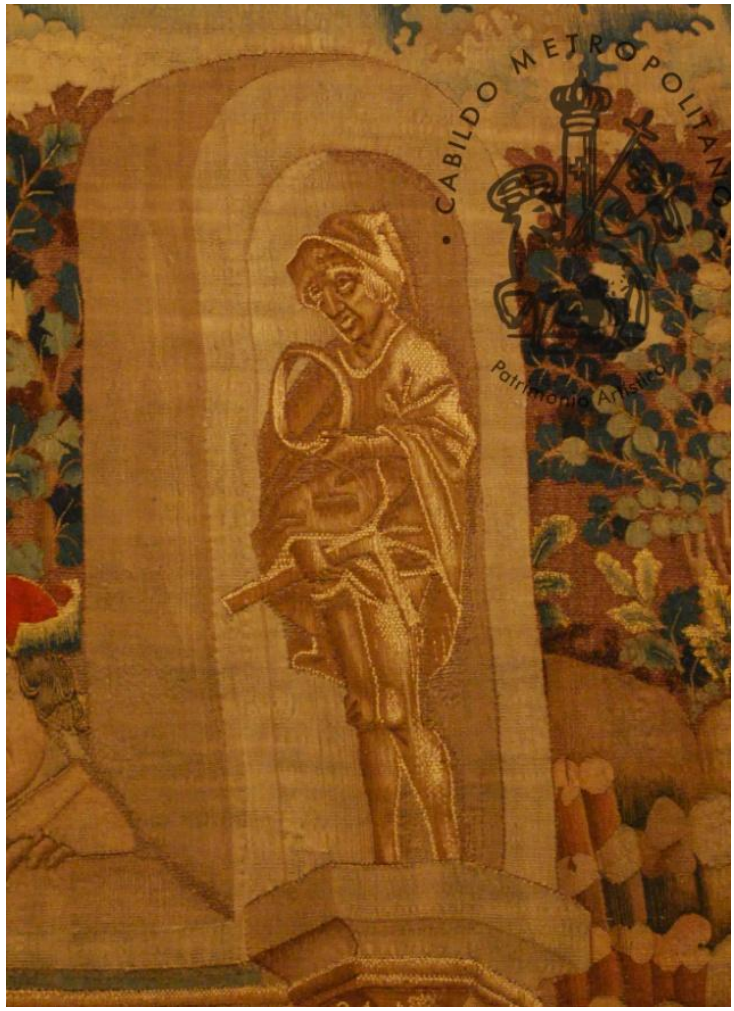

Fig. 9. Detalle del Tapiz de "Cautividad de la Santa Cruz". Fotografía cedida por cortesía del Museo de Tapices. Cabildo Metropolitano de Zaragoza.

caso, en vez de un aro un compás. Esta figura, pues, representaría a alguien encargado de medir.

La figura de la izquierda (Fig. 10) es la que nos interesa. La figura representa a un hombre ataviado con un peto o túnica y un sombrero de copa cubriéndole la cabeza. Sujeta entre sus manos un objeto de gran longitud (más largo que su propio brazo) que parece ser un tubo de mirada. Aunque no es descartable que el objeto no sea un tubo hueco sino más bien una tabla u otro elemento compuesto de una superficie plana. En cualquier caso, aunque la naturaleza del objeto, bien sea un tubo hueco o una superficie plana, es un tanto ambigua, no así su función. Nuestro personaje sujeta el instrumento con ayuda de ambas manos apoyándolo sobre su cara de manera que ve con su ojo izquierdo en la dirección marcada por el instrumento. El instrumento le sirve, pues, para dirigir la mirada hacia la figura del rey Cosroes. Tal como hemos introducido previamente un instrumento que cumple esta función se puede calificar de dioptra.

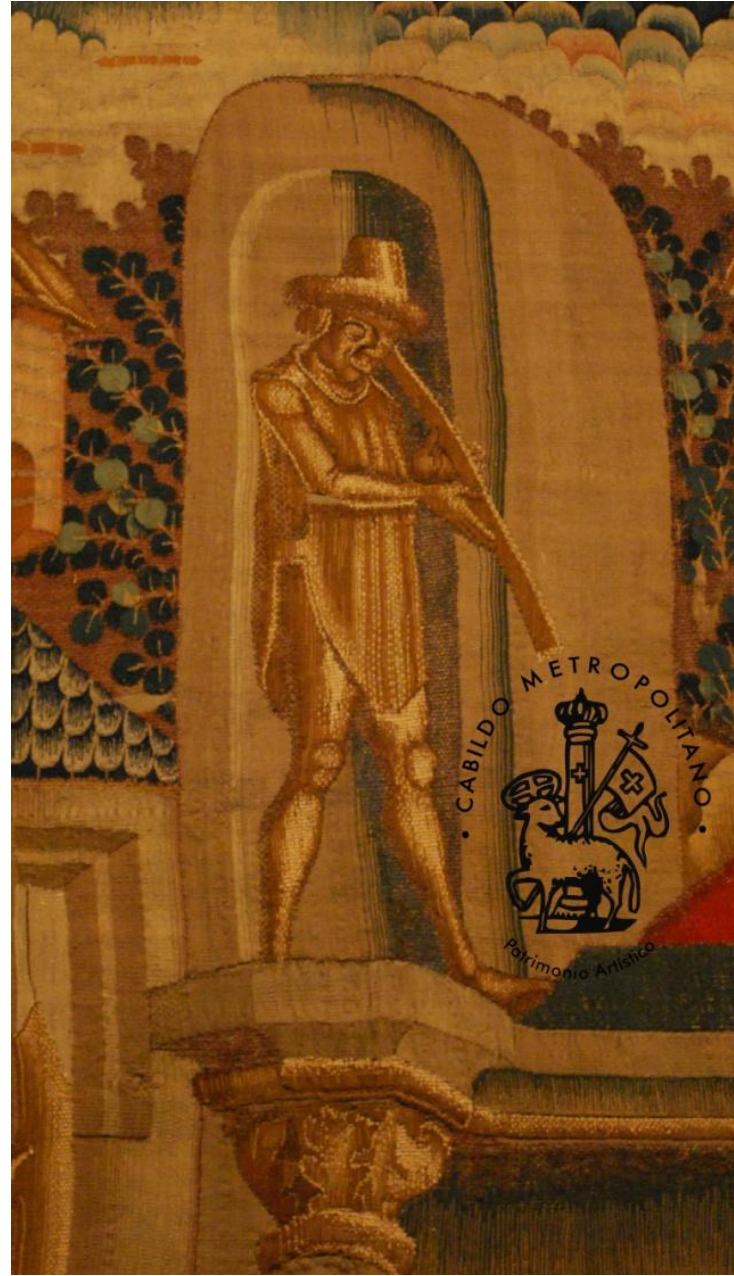

Fig. 10. Detalle del Tapiz de "Cautividad de la Santa Cruz". Fotografía cedida por cortesía del Museo de Tapices. Cabildo Metropolitano de Zaragoza.

Resulta significativo el hecho de que la dioptra aparece ligeramente quebrada, de manera que no marca una línea recta. A mi entender, y avalado por la opinión de un responsable del Museo de Tapices, la explicación más razonable de este peculiar efecto es que es debido a la deformación del tapiz producida por el transcurso del tiempo. Esta hipótesis se sustenta porque en otras escenas del tapiz se pueden observar diversos contornos, que debiendo ser líneas rectas, también aparecen curvados. Es por ejemplo el caso de las columnas adyacentes al trono de Cosroes (Fig. 8). Estas aparecen curvadas, en especial la columna de la izquierda. El tapiz, extremadamente pesado debido a sus dimensiones, se sujeta en su parte superior a una cuerda con ayuda de velcro. Este sistema de sujeción sustituye a uno anterior 
basado en cincha y cuerda. Por lo tanto, la fuerza de la gravedad, y otros condicionantes físicos, a lo largo del tiempo pueden deformar la estructura de trama y urdimbre del tapiz. Esto explica las distorsiones que se ven en la representación de la escena.

Dentro de la clasificación iconográfica descrita en la sección tercera, la dioptra no parece ser, en primera instancia, de la clase de instrumento de observación astronómica, sino más bien de los usados con otros fines, ya que nuestro personaje dirige la mirada al rey Cosroes en vez de al cielo. Sin embargo un análisis conceptual más detallado puede conducirnos a una reinterpretación. Queda patente, por los textos reproducidos, que la tradición cristiana, en su crítica al rey Cosroes, subraya el afán de éste por ser adorado como dios. Así en la inscripción del tapiz se dice: "queriendo ser adorado como dios», o en la Leyenda Dorada: «en su afán de que sus súbditos le tuvieran por dios». La asociación de los astros con divinidades ha existido tanto en la cultura oriental como occidental antigua. Valga como recordatorio que así lo pensaban los pitagóricos, que influyeron a Platón en su idea de los «dioses visibles» presente en su diálogo Timeo. De hecho algunos de los primeros filósofos de la Antigüedad Clásica, Anaxágoras y Socrates, fueron acusado de impíos (contrarios a la religión) por sostener que el sol y la luna no eran dioses sino piedras. Si a los astros, divinidades, se les mira con ayuda de una dioptra, Cosroes en su afán de mostrarse como un Astro-Divinidad, querría ser visto de la misma manera. Por lo que nuestro personaje, que sostiene la dioptra, aparece representado en pequeño para dar la sensación de lejanía. El súbdito del rey Cosroes, observa a este, desde lejos, como si fuese un Astro (ser divino), y por tanto con la ayuda de una dioptra.

\section{Agradecimientos}

Agradezco a la Dirección de Patrimonio Artístico del Cabildo Metropolitano de Zaragoza por realizar y permitir la publicación de las fotos correspondientes a las Figs. 8, 9 y 10. Agradezco de manera especial la ayuda de Juan Antonio Montolío Palacín por la información aportada sobre la historia del tapiz "Cautividad de la Santa Cruz". Por último agradezco los valiosos comentarios de uno de los revisores anónimos de este artículo. 